Planetary Systems in the Universe - Observation, Formation and Evolution Proceedings IAU Symposium No. 202, (C)2004 IAU

Alan Penny, Pawel Artymowicz, Anne-Marie Lagrange, $\mathcal{G}$ Sara Russell, eds.

\title{
Are there Jupiters in the $\alpha$ Centauri system?
}

\author{
Michael Endl
}

1. European Southern Observatory, Casilla 19001, Santiago, Chile

2. Institut für Astronomie, Universität Wien, Türkenschanzstr. 17, A-1180 Wien, Austria

Martin Kürster

European Southern Observatory, Casilla 19001, Santiago, Chile

\section{Sebastian Els}

1. European Southern Observatory, Casilla 19001, Santiago, Chile

2. Institut für Theoretische Astrophysik, Universität Heidelberg, Tiergartenstr.15, D-69121 Heidelberg, Germany

Artie P. Hatzes

Thüringer Landessternwarte Tautenburg, Sternwarte 5, D-07778 Tautenburg, Germany

William D. Cochran

McDonald Observatory, The University of Texas at Austin, Austin, $T X$ 78712-1083, USA

\section{Konrad Dennerl}

Max-Planck-Institut für extraterretrische Physik, Giessenbachstr., D-85748 Garching, Germany

Stefan Döbereiner

Max-Planck-Institut für extraterretrische Physik, Giessenbachstr., D-85748 Garching, Germany

\footnotetext{
Abstract. We present the results of five years of high precision radial velocity (RV) monitoring of the $\alpha$ Centauri system. Observations were performed with the ESO 1.4m CAT telescope and the Coude Echelle Spectrometer (CES) on La Silla. From the achieved RV precision we establish the detection threshold for Jovian planets in orbit around $\alpha$ Centauri A \& B. Together with the known dynamical limits for stable planetary orbits we can set strong constraints on the presence of giant planets in our neighbouring binary system.
} 


\section{Observations and Radial Velocities}

The $\alpha$ Centauri system has been monitored for radial velocity (RV) variations since Nov. 1992 by the planet search program carried out at the ESO Coudé Echelle Spectrometer (CES) on La Silla (Kürster et al. 2000). A detailed description of the instrument setup, the observational process, and the data analysis technique of the CES planet search can be found in Endl et al. (2000). Until April 1998 we obtained a total of 48 RV measurements (nightly means) of $\alpha$ Centauri A and 43 of component B respectively. From these RV results we subtracted the known binary orbital motion given by Pourbaix et al. (1999). The residual RV scatter (RMS) around this orbit is $11.8 \mathrm{~ms}^{-1}$ for component $\mathrm{A}, 12.6 \mathrm{~ms}^{-1}$ for component $\mathrm{B}$, and reveals no significant periodic signal.

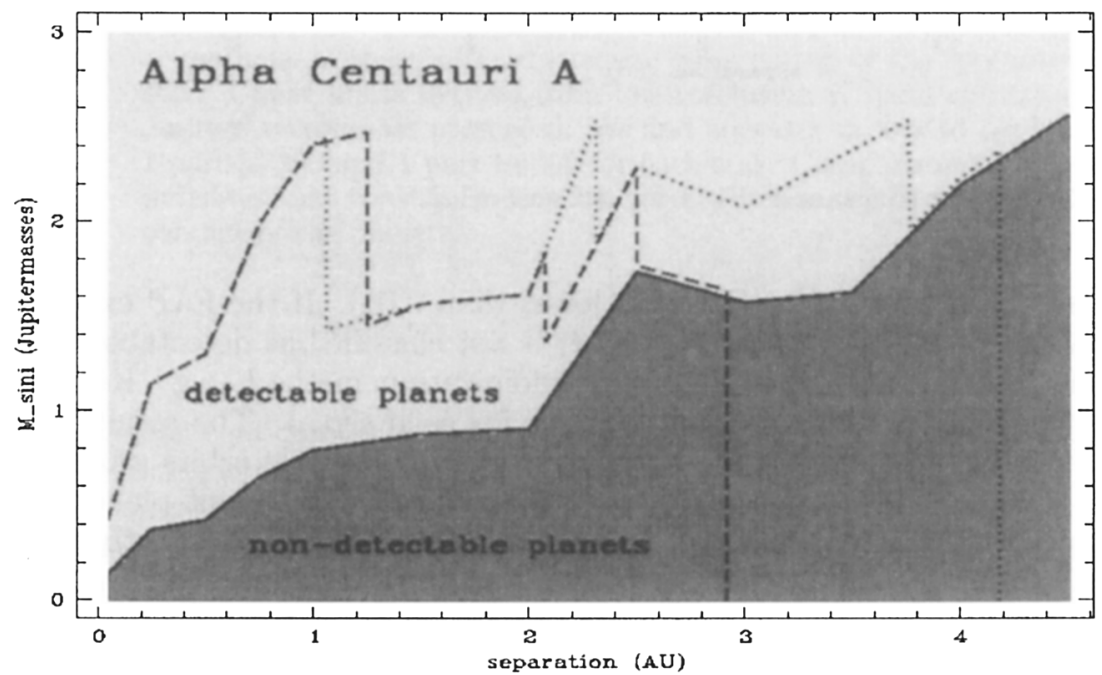

Figure 1. Upper limits for planets orbiting $\alpha$ Centauri A. The y-axis gives the projected mass $(M \sin i)$ of the planet, with $i$ as the viewing angle to the planetary orbit. Planets orbiting in the binary plane $\left(i=79.23^{\circ}\right)$ would have masses only slightly larger than their $M \sin i$ indicated by the solid line $\left(i=90^{\circ}\right)$. The other two lines show the dynamical limits for stable prograde (dashed line) and retrograde (dotted line) orbits given by Wiegert \& Holman (1997), where orbits with smaller separations remain stable also at higher inclinations.

\section{Limits for Planetary Companions}

Starting from the null - hypothesis that this residual scatter is caused only by measurement uncertainties, we establish the detection threshold in order to obtain upper limits for giant planets in the $\alpha$ Centauri system. We create simulated signals of planets in circular orbits with varying masses (amplitudes), orbital phases and periods (separations). These signals are added to the actual 


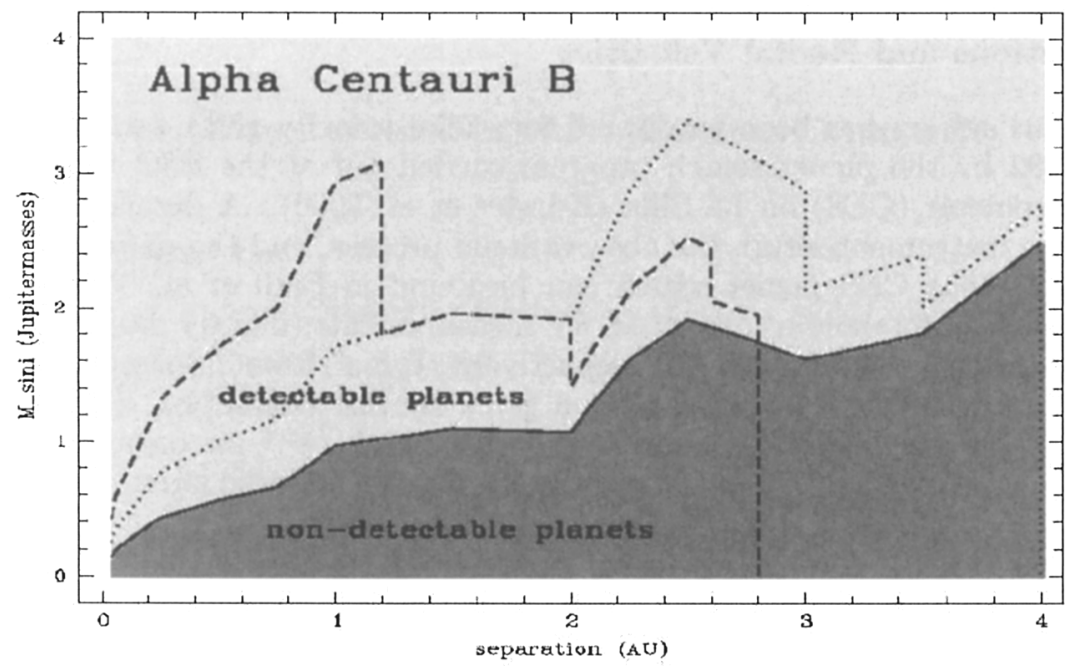

Figure 2. Same diagram as Fig.1 for $\alpha$ Centauri B.

(i.e. its false alarm probability (FAP) is lower than 0.01). If the FAP exceeds 0.01 at only one of the trial phases, a planet is not classified as detectable. We estimate the FAP by using a bootstrap randomization method (e.g. Kürster et al. 1996) and perform 1000 bootstrap runs for each signal. The results are shown in Fig.1 \& 2, along with the dynamical limits for stable orbits given by Wiegert \& Holman (1997). For $\alpha$ Centauri A we can exclude giant planets in circular orbits with projected masses larger than 1 or $2.5 M_{\mathrm{Jup}} \sin i$ (with $i$ the orbital inclination) at orbital radii less than 2 and $4.5 \mathrm{AU}$, respectively. For component B we exclude planets with 1 (2.5) $M_{\mathrm{Jup}} \sin i$ at 1.1 (4) AU.

Acknowledgments. We thank the ESO OPC for generous allocation of observing time to the CES planet search. M. Endl acknowledges support by the Austrian Fond zur Förderung der wissenschaftlichen Forschung Nr. S7302.

\section{References}

Endl, M., Kürster, M., \& Els, S. 2000, A\&A, in press.

Kürster, M., Endl, M., Els, S., Hatzes, A.P., Cochran, W.D., Döbereiner, S., Dennerl, K. 2000, A\&A, 353, L33

Kürster, M., Schmitt J.H.M.M., Cutispoto G., Dennerl, K. 1996, A\&A, 320, 831

Pourbaix, D., Neuforge-Verheecke, C., \& Noels, A. 1999, A\&A, 344, 172

Wiegert, P.A., \& Holman, M.J. 1997, AJ, 113 (4), 1445 